\title{
OPTICAL AND X-RAY VARIABILITY IN NGC 4395
}

\author{
P. LIRA AND A. LAWRENCE \\ Institute for Astronomy, University of Edinburgh, \\ Royal Observatory, Blackford Hill, Edinburgh EHg зHJ, UK
}

\section{NGC 4395, The Least Luminous Seyfert 1 Galaxy}

The spectrum of the dwarf Seyfert 1 nucleus in NGC 4395 was first reported about a decade ago (Filippenko \& Sargent 1989), showing high ionization narrow emission lines and broad permitted lines. The later detection of radio and X-ray compact nuclear sources and of a featureless UV continuum gave support to the idea that NGC 4395 is a genuine low luminosity AGN (Sramek 1992 ; Filippenko, Ho \& Sargent 1993). However, it has been claimed that, unlike classic Seyferts, NGC 4395 does not vary at all (Shields \& Filippenko 1992).

\section{Optical and X-ray Observations}

As part of a multiwavelength and spectroscopic study of a sample of nearby galaxies, we obtained optical long-slit spectra of NGC 4395 on July 1996 and January 1997 at the WHT. No variation in the narrow-line fluxes exceeds the spectrophometric errors. The data also indicate that the NLR in NGC 4395 is spatially unresolved, so scaling to the narrow-lines gives us a relative flux calibration accurate to a few percent. As can be seen in Fig 1 the nucleus of NGC 4395 varied by a factor of two within six months, becoming bluer when brighter. A smaller amplitude change in the broad $\mathrm{H} \alpha$ component $(\sim 30 \%)$ is observed.

A ROSAT HRI image of NGC 4395 was obtained in June 1996. A weak source consistent with the nucleus of NGC 4395 was identified, although the low count rate prevents us from claiming a robust detection. Two ROSAT PSPC images retrieved from the public archive showed that in July 1992 an X-ray source, spatially consistent with our HRI detection, varied by a factor of $\sim 2$ in 15 days. From these three ROSAT observations we find for NGC 4395 a weighted mean X-ray flux of $1.2 \pm 0.1 \times 10^{-13} \mathrm{ergs} \mathrm{s}^{-1} \mathrm{~cm}^{-2}$. 


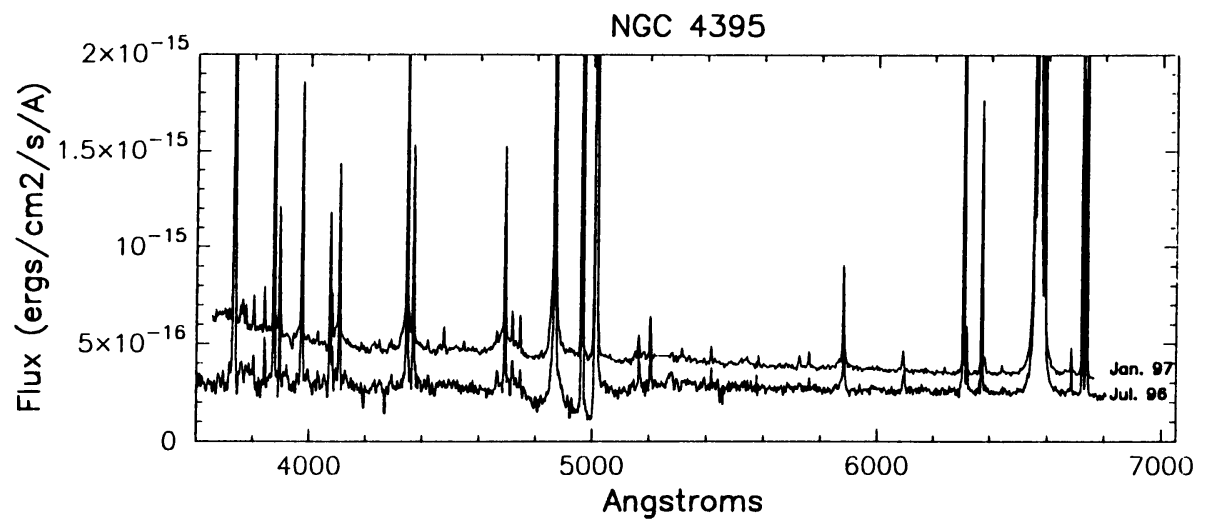

Figure 1. Optical spectra showing high and low state of NGC 4395.

\section{The Starbust Hypothesis}

We can rule out the possibility that the spectrum of NGC 4395 is produced by an ordinary stellar population since no stellar features are seen in the optical or UV spectra. It has been suggested that a single SNR (just one supernova is enough to explain the NGC 4395 luminosity $-M_{B}^{N u c} \sim-10$ ), expanding in a dense circumstellar medium, could explain the nuclear emission, since the broad $\mathrm{H} \alpha$ would not be expected to vary over time scales of a few years (Filippenko 1992). However, our observations show that the continuum and broad emission have increased over a 6 months period, which is not compatible with the evolution of a SNR event.

\section{Summary}

We have shown that the nucleus of NGC 4395 does vary, with the optical continuum changing in amplitude as well as in colour, with broad line flux variations of $\sim 30 \%$, and with X-ray flux changes by a factor of 2 .

The detection of variability, together with all the previous evidence, implies that low luminosity nuclear activity does not just mimic classical AGN in particular features, but that the same (scaled) physical mechanisms are required to explain it.

\section{References}

Filippenko, A.V., 1992, in Relationships Between Active Galactic Nuclei and Starbust Galaxies, p. 253, A.S.P. Conf. Ser., Vol 31, Filippenko, A.V. (ed.)

Filippenko, A.V. \& Sargent, W.L., 1989, Ap. J. (Letters), 342, L11

Filippenko, A.V., Ho, L.C. \& Sargent, W.L., 1993, Ap. J. (Letters), 410, L75

Shields, J.C. \& Filippenko, A.V., 1992, in Relationships Between Active Galactic Nuclei and Starbust Galaxies, p. 267, A.S.P. Conf. Ser., Vol 31, Filippenko, A.V. (ed.)

Sramek, R., 1992, in Relationships Between Active Galactic Nuclei and Starbust Galaxies, p. 273, A.S.P. Conf. Ser., Vol 31, Filippenko, A.V. (ed.) 